COLLOQUUIUM MATHEMATICUM

\begin{tabular}{lll}
\hline VOL. III & 1955 & FASC. 2 \\
\hline
\end{tabular}

$\begin{array}{llllllllllllll}\mathrm{C} & \mathrm{O} & \mathrm{M} & \mathrm{M} & \mathrm{U} & \mathrm{N} & \mathrm{I} & \mathrm{C} & \mathrm{A} & \mathrm{T} & \mathrm{I} & \mathrm{O} & \mathrm{N} & \mathrm{S}\end{array}$

ON A NEW ANALYTICAL MIETHOD AND ITS APPLICATIONS*) $\mathrm{BY}$

P. TURAN (BUDAPEST)

The theory of diophantine approximation has played a part in the analysis and in the theory of numbers for a long time. Two theorems of that theory are mainly responsible for it.

I. Theorem of Dirichlet. Given $n$ positive numbers $\alpha_{1}, a_{2}, \ldots, a_{n}$ and an integer $q \geqslant 3$ we can find a $t$-value in $1 \leqslant t \leqslant q^{n}$ for which the $t \alpha_{v}$-valnes are all nearer to a rational integer than $1 / q$.

If $\{x\}$ denotes the distance of the real number $x$ from the next integer, then it follows that for an arbitrarity small positive $\varepsilon$ there is a real $t_{0}$ such that

$$
\left\{t_{0} a_{v}\right\} \leqslant \varepsilon
$$

$(p=1,2, \ldots, n)$

and

$$
1 \leqslant t_{0} \leqslant\left(\frac{1}{\varepsilon}\right)^{n}
$$

II. Theorem of Kroneeker. Given $n$ positive linearly independent $\alpha_{1}, a_{2}, \ldots, \alpha_{n}$, the real numbers $\beta_{1}, \beta_{2}, \ldots, \beta_{n}$ and a positive arbitrarily small $\varepsilon$, then there is a real $t_{1}$ strch that $\left\{t_{1} \alpha_{v}-\beta_{v}\right\} \leqslant \varepsilon(v=1,2, \ldots, n)$.

Here no limitations for $t_{1}$, such as (2), can be given. Linear independence means that no relation $r_{1} a_{1}+r_{2} a_{2}+\ldots+r_{n} a_{n}=0$ can hold with rational $r_{v}$ except if $r_{1}=r_{2}=\ldots=r_{n}=0$.

To give an idea of the applicability of these threorems we mention some examples. The first and simplest is of arithmetical nature and refers to the non-trivial solubility of the Pell-equation

$$
x^{2}-d y y^{2}=1
$$

(d positive non-square integer; $x= \pm 1, y=0$ are the trivial solutions). An important step in the classical proof of Lagrange is the proof of the

\begin{tabular}{|c|c|}
\hline $\begin{array}{l}\text { Naktad } 1595+25 \text { egz.. } \\
\text { Ark. wyds. 8,75; druk. } 8 \\
\text { Papier druk, sat. bezdkz. } 70 \times 100,100 \mathrm{~g} \\
\text { Cema zl 17,50 }\end{array}$ & 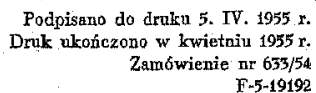 \\
\hline
\end{tabular}
solubility of the inequality

$$
|\mu \sqrt{d}-\vartheta|<\frac{1}{\mu}
$$


with integer $\mu>1$ and $\vartheta$, which follows easily from Dirichlet's theorem for $n==1$. The second example is of function-theoretical nature, due to H. Bohr, and refers to the question, raised by Lindelof, whether or not the zeta-function of Riemann is bounded in the domain $\sigma>1,|t| \geqslant 1$ of the complex variable $s=\sigma+i t$. This funotion - introduced by Riemann in 1859 for the study of the distribution of primes - is defined in this half-plane by. the series

$$
\zeta(s)=\sum_{n=1}^{\infty} \frac{1}{n^{s}}
$$

By a very ingenious, completely elementary proof, Bohr answered lindelof's question in the negative. Obviously it suffices to show that for an arbitrary large positive $\omega$ there is an $s_{1}=\sigma_{1}+i t_{1}$ with $\sigma_{1}>1$ and

$$
R \zeta\left(s_{1}\right)=1+\sum_{n=2}^{\infty} \frac{\cos \left(t_{1} \log n\right)}{n^{\sigma_{1}}}>\omega .
$$

To show this Bohr wrote first

$$
R \zeta(s)>1+\sum_{n=2}^{N} \frac{\cos (t \log n)}{n^{\sigma}}-\sum_{n=N+1}^{\infty} \frac{1}{n^{\sigma}}
$$

The first sum is estimated at a fixed $N$ from below for a suitable choice of $t$. To do this he applied Dirichlet's theorem with

$$
\frac{1}{q}=\varepsilon=\frac{1}{5}, \quad a_{y}=\frac{1}{3 \pi} \log \nu \quad(v=2,3, \ldots, N) .
$$

Choosing the resulting $t_{0}$ as $t_{1}$ we have

$$
\cos \left(t_{2} \log v\right) \geqslant \cos \frac{2 \pi}{5} \quad(y=3,3, \ldots, N)
$$

where for $t_{1}$

$$
1 \leqslant t_{1} \leqslant 0^{N-1}
$$

holds. Hence

$$
\begin{aligned}
R \zeta\left(\sigma+i t_{1}\right) & >\cos \frac{2 \pi}{5}\left(\frac{1}{1^{\sigma}}+\frac{1}{2^{\sigma}}+\ldots+\frac{1}{N^{\sigma}}\right)-\sum_{n=N+1}^{\infty} n^{-\sigma} \\
& >\cos \frac{2 \pi}{5} \sum_{n=1}^{\infty} n^{-\sigma}-2 \sum_{n=N^{N}+1}^{\infty} n^{-\sigma}>\left(\frac{1}{2}-\frac{2}{(N+1)^{\sigma-1}}\right) \frac{1}{\sigma-1}
\end{aligned}
$$

as the integral-criterion shows at once. Now fixing $\sigma=\sigma_{1}$ with $\sigma_{1}=1+1 / 4 \dot{\omega}$ we choose $N$ so large that

$$
\frac{2}{(N+1)^{\sigma^{1-1}}}<\frac{1}{4}
$$

Then, ehoosing $t_{I}$ as in (5), Bohr obtained

$$
R \zeta\left(\sigma_{1}+i t_{1}\right)>\frac{1}{1} \cdot \frac{1}{\sigma_{1}-1}=\omega
$$

i.e. $\zeta(s)$ assumes indeed arbitrarily large values in the domain $\sigma>1$, $|t| \geqslant 1$. Usually we express growth as a function of $t$; using in an essential manner the limitation (5), we obtain also

$$
R \zeta\left(\sigma_{1}^{\prime}+i t_{1}^{\prime}\right)>\frac{1}{9} \log \log t_{1}^{\prime} \quad\left(t_{1}^{\prime} \text { arbitrariiy big, } \sigma_{1}^{\prime}>1\right) .
$$

Both former applications used Dirichlet's theorem. To see one of the numerous applications of Kronecker's theorem, we consider the amalogous question concerning $1 / 5(s)$. We have for $\sigma>1$

$$
\frac{1}{\zeta(s)}=\sum_{n=1}^{\infty} \frac{\mu(n)}{m^{s}}
$$

where $\mu(n)$ stauds for Möbius' number-theoretical function, $i . e . \mu(n)=0$ if $n$ has a quadratic factor greater than 1 and $(-1)^{r}$ if $n$ is built up from $r$ different prime-factors. As Bohr showed, a similar reasoning - only using Kronecker's theorem instead of Dirichlet's - shows also that $1 / \zeta(s)$ assumes arbitrarily large values in the half-plane $\sigma>1$. This, in 1911, was a rery surprising result since it means that $\zeta(s)$ assumes for $\delta>1$ arbitrarily small values, whereas the famous conjecture of Riemann, so far unproved, asserts that all the zeros of $\zeta(s)$ lie in the half-plane $\sigma \leqslant 1 / 2$.

What is the essence of the preceding proofs? In both cases we had to estimate trigonometrical sums of the form

$$
\sum_{\nu=1}^{N} a_{n} e^{i l \log v}
$$

for suitable $t$-values from below by $\sum_{p=1}^{N}\left|a_{p}\right|$. Applications to general Dirichlet-series raise an amalogous question for sums of the form

$$
1+\sum_{n=2}^{N} a_{n} e^{2 \pi i a_{n} t} \quad\left(a_{1}<a_{2}<\ldots<\alpha_{n}\right) .
$$


Now Dirichlet's theorem holds again for the casc when $a_{s}>0$ and $a_{1}<\alpha_{2}<\ldots$; and Kronecker's theorem holds if the $a_{\nu}$ aro linearly independent and the $a_{v}$-coefficients are arbitrary. In the first caso Diriohlet's theorem gives at once the existence of a $t^{\prime}$ such that for an arbitrary $0<\varepsilon<1 / 5$

$$
1 \leqslant t^{\prime} \leqslant\left(\frac{1}{\varepsilon}\right)^{n},
$$

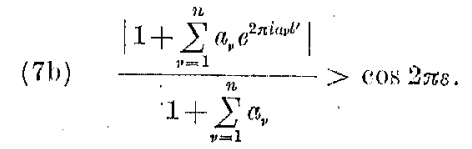

In the second case Kronecker's theorem gives for an arbitrary positive $\varepsilon$ smaller than, say, $1 / 4$ a real $t^{\prime \prime}$ such that

$$
\frac{\left|1+\sum_{\nu=1}^{n} a_{y} e^{2 \pi i u p t^{\prime \prime}}\right|}{1+\sum_{\nu=1}^{n}\left|a_{y}\right|}>1-\varepsilon
$$

but without any upper limitation for $t^{\prime \prime}$.

The solubility of (7) and (8) is a simple consequence of the theorems of Dirichlet and Kronecker respectively. What can be said conversely ? First we consider the relationship of (8) and Kronecker's theorem more closely. We fix in (8) the positive $\left|a_{y}\right|$-values and the linearly-independent $a_{w}$-system. Then, if the trigonometrical inequality (8) has a solution in $t$ for an arbitrarily small positive $\varepsilon$, we can easily deduce Kronecker's theorem for the same $\left(a_{p}, a_{w}\right)$-system. Hence, as to their content, the solubility of the trigonometrical inequality (8) and the theorem of Kronecker are equivalent. The solubility of (8) can be proved also independently of Kronecker's theorem. This indeed has been done in essence by Bohr. In the same way one can show more generally that Kronecker's theorem is equivalent to the fact that for any special positive $\left|a_{y}\right|$-system and a system of $z_{1}, z_{2}, \ldots, z_{n}$ complex numbers with linearly independent arguments and arhitrarily small positive $\varepsilon$ the inequality

$$
\frac{\left|1+\sum_{i=1}^{n} a_{v} z_{y}^{t}\right|}{1+\left.\sum_{j=1}^{N}\left|r_{v}\right| ! z_{p}\right|^{t}}>1-\varepsilon
$$
has a real $t$-solution. Of course the condition $\left|z_{j}\right|=1$ is no longer requi-
red.

The relationship between Dirichlet's theorem and the simultaneous solubility of the inequality (7) is more complicated. The difference is caused by the fact that Kroneeker's theorem is always stated only for arbitrurily small $\varepsilon$ while Dirichlet's theorem in the usual form is stated for all $0<\varepsilon$ not larger than, say, $1 / 5$, and not, as we did in (1)-(2), for "small" $\varepsilon$ only. The reason for that lies obviously in the limitation (2). Nevertheless, for most applications in the analysis the form (1)-(2) suffices; we confine ourselves to the comparison of (1)-(2) with (7) if the positive $a_{v}$ and $\alpha_{y}$ are fixed and $\varepsilon$ is small. In the same way as before we can show that they are also equivalent. As to a proof of (7), independent of Dirichlet's theorem, it is highly probable that this can be done; I have beon able to prove it for special $a_{\nu}$-sequences like $\alpha_{\nu}=\log \nu$, and probably the general case can be settled in this way after some changes. That would be very desirable; it would prove that the solubility of (7) is "essentially" equivalent; to the form (1)-(2) of Dirichlet's theorem. The connection between Kroneoker's theorem and its trigonometrical form (see (9)) has its counterpart in the following relation: the form (1)-(2) is "essentially" equivalent to the fact that for any special positive $a_{4}$-system, for any system $z_{1}, \ldots, z_{n}$ of complex numbers and for all sufficiently small $\&$ the inequality

$$
\frac{\left|1+\sum_{v=1}^{n} a_{p} z_{v}^{t}\right|}{1+\sum_{\nu=1}^{n} a_{p}\left|z_{p}\right|^{t}}=\frac{\left|1+\sum_{v=1}^{n} a_{p}^{i} z_{\nu}^{l}\right|}{1+\sum_{\nu=1}^{n} a_{p}\left|z_{\nu}\right|^{t}}>\cos 3 \pi \varepsilon
$$

has a solution satisfying the condition

$$
1 \leqslant t \leqslant\left(\frac{1}{\varepsilon}\right)^{n}
$$

"Essentially equivalent" means that from (10) we can deduce the inequality (1)-(2) only in a somewhat weaker form, replacing $\varepsilon$ in (1) by another function of $\varepsilon$ which tends to 0 with $\varepsilon$.

The inequalities (9) and (10) both have a very similar form; both estimate under special conditions the "power-sums"

$$
\left|1+\sum_{\nu=1}^{n} a_{v} z_{\nu}^{t}\right|
$$

from below for special $t$-values, by

$$
1+\sum_{y=1}^{n}\left|\omega_{y}\right| z_{y}||^{\prime}
$$

The localisation of the $t$-valnes accomplishing this is either impossible or inexact but the range of this t-value depends only upon $\varepsilon$; however, in many problems of analysis - as we shall see - a much better localisation would be necessary even at the cost of sacrificing something of the strength of the lower estimation. 
In fact, the lower estimation is very strong if we take into account that for all $t$-values

$$
\frac{\left|1+\sum_{\nu=1}^{n} a_{\nu} z_{\nu}^{t}\right|}{1+\sum_{\nu=1}^{n}\left|a_{\nu}\right|\left|z_{\nu}\right|^{t}} \leqslant 1 .
$$

The first question was to find appropriate expressions - which may be called norms - for the lower estimation. In a systematio study of this question I found that besides the expression

$$
1+\sum_{i=1}^{n}\left|a_{v}\right|\left|z_{y}\right|^{t}
$$

the Bohr norm, also three other norms play an important role. These are, if $z_{0}=1$,

$$
\begin{gathered}
M_{1}(t)=\left(\max _{j=0,1, \ldots, n}\left|z_{j}\right|\right)^{t}, \quad M_{2}(t)=\left(\min _{j=0,1, \ldots, n}\left|z_{j}\right|\right)^{t}, \\
\quad M_{3}(t)=\left(1+\sum_{j=1}^{n}\left|a_{n}\right|^{2}\left|z_{v}\right|^{2 t}\right)^{1 / 2},
\end{gathered}
$$

the last may be ealled, for certain reasons, $N$. Wiener's norm. It is not impossible that in the course of research the necessity of other norms will also emerge. Another important observation was that the lower estimation can be allowed to depend also upon the coefficients $a_{v}$ in a reasonable way; only it should be independent of the configuration of the $z_{j}{ }^{3}$ ). At the cost of these concessions I have been able to prove two lower estimations of $\left|\sum_{v=1}^{N} b_{\nu} z_{\nu}^{t}\right|$ by the norms $M_{1}(t)$ and $M_{2}(t)$ which hold for a "sufficiently" dense set of $t$-values and without any restriotion upon the $b_{w}$-coefficients and $z_{z}$-numbers. The two main theorems I have obtained are as follows:

THEOREM I. If $n$ is an arbitrary positive number, then the inequality

$$
\frac{\left|\sum_{p=1}^{n} b_{p} t_{\nu}^{b}\right|}{\lambda U_{2}(t)} \geqslant\left(\frac{n}{6(n+n)}\right)^{n}\left|\sum_{p=1}^{n} b_{p}\right|
$$

has an integer-solution $t=t_{2}$ sivch that $m \leqslant t_{2} \leqslant m+n$.

Another, sometimes more convenient form of this theorem is the following

1) Added in proof. Since that time I realized the importance of lower estima. tions independent of the $a_{1}$ coefficients only. In my book entitled Utber eine nere Methode in der Analysis und deren Anwendungen printed in 1953 I did not realize it fully, though this book contains results in that direction already.
THEOREM Ia. If $0<a<b$, then the inequality

$$
\frac{\left|\sum_{\nu=1}^{n} b_{\nu} z_{\nu}^{t}\right|}{M_{2}(t)}>\left(\frac{b-a_{1}}{3 e b}\right)^{n}\left|\sum_{\nu=1}^{n} b_{\nu}\right|
$$

has a solution $t=t_{3}$, with $a \leqslant t \leqslant b$. Here $z_{y}^{t}$ means $e^{t \log t \mathrm{t}}$ with an arbitrarily fixed values of the logarithm.

The second, main theorem refers to $M_{1}(t)$ and is of course deeper.

THEOREMI II. If $m$ is an arbitran'y positive number and if $\left|z_{1}\right| \geqslant\left|z_{2}\right| \geqslant \ldots$ $\geqslant\left|\hat{z}_{2}\right|$, then the inequatity

$$
\frac{\left|\sum_{\nu=1}^{n} b_{\nu} z_{v}^{t}\right|}{M_{1}(t)} \geqslant\left(\frac{n}{250(m+2 n)}\right)^{n} \min _{j=1,2, \ldots, n}\left|b_{1}+b_{2}+\ldots+b_{j}\right|
$$

has an integer solwtion $t=t_{4}$ for which $m \leqslant t_{4} \leqslant m+n$.

The proofs of these theorems are elementary though somewhat artificial; this refers in particular to that of theorem II.

Here is a sketch of the proof of theorem I. It is easy to reduce the proof to the case when $M_{2}(t)=1$ and $m$ is a positive integer. Then we form the amxiliary polynomial

$$
f_{1}(z)=\prod_{j=1}^{n}\left(1-\frac{z}{z_{j}}\right)=\sum_{l=0}^{n} c_{l} z^{l}
$$

and the power-series

$$
\frac{1}{f_{1}(z)}=\sum_{i=0}^{\infty} d_{l} z^{l}
$$

which converges for $|z|<1$; the absolute value of the coefficients $o_{2}$ and $a_{l}$ can be estimated easily from above. Forming the further auxiliary polynomial

$$
f_{2}(z)=1-f_{1}(z) s_{m}\left(\frac{1}{f_{1}}\right)=\sum_{l m 0}^{m+n} d_{l}^{\prime} z^{l}
$$

of degree $m+n$ (where $s_{m}\left(1 / f_{1}\right)$ denotes the $m$-th partial sum of $1 / f_{1}(z)$ ), we may observe first that the coefficients $d_{0}^{\prime}, d_{1}^{\prime}, \ldots, d_{m}^{\prime}$ vanish, further that $f_{2}(z)$ assumes the value 1 for $z=z_{1}, z=z_{2}, \ldots, z=z_{n}$ and finally, that the absolute value of the remaining coefficients $d_{l}^{\prime}$ can easily be estimated from above. Hence, replacing in (11) $z$ by $z_{i}$, multiplying by $b_{j}$, and summing for $j=1,2, \ldots, n$, we obtain the identity

$$
\sum_{i=m+1}^{m+n} a_{l}^{\prime}\left(\sum_{j=1}^{n} b_{j} z_{j}^{l}\right)=\sum_{j=1}^{n} b_{j},
$$


from which theorem I follows at once if the estimations for $\left|d_{2}^{\prime}\right|$ are used. The proof of theorem II is based on similar ideas but is much more elaborate. Extensive use is made of the well-known theorem of H. Cartan, according to which for a given polynomial $g(z)$ of degree $N$ and a given. positive $H$ the inequality

$$
|g(z)| \geqslant\left(\frac{H}{e}\right)^{N}
$$

holds on the whole complex plane exoept a set which can be covered by at most $N$ circles with the radius-sum $2 H$ and of an integral-representation of the coefficients of the Newton interpolation formula due to Nör$\operatorname{lun} \mathrm{d}^{2}$ ).

The applicability of these theorems is indicated already by the fact that they estoimate a sum from below essentially by its minimal or maximal terms, practically without restrictions, which could not be said either of Kronecker's or of Dirichlet's theorem. The advantages of much better localisation will be clear from the applications. The dependence upon the coefficients, as regards simplicity, leaves still much to be desired, particularly in the case of theorem II. This is the reason why the applications of this theorem are confined to the case of positive $b_{i}$, where the critical factor can be replaced simply by $\min b_{j}=\min \left|b_{j}\right|$. To remove this obstacle would be an important question of the theory. The hope that expressions on the right in theorems I and II can be replaced generally by $\max \left|b_{j}\right|$ or even by $\min \left|b_{j}\right|$ is removed by the simple counter. -example

$$
b_{1}=1, \quad b_{2}=b_{3}=\ldots=b_{n}=-\frac{1}{n-1}, \quad z_{1}=\approx_{2}=\ldots=z_{n}=1 .
$$

It would be interesting to find another form of theorem $I$, where the right side depends only upon min $\left|b_{j}\right|$, even at the cost of imposing further simple geometrical restrictions upon the $z_{y}$, for it would also have applications. Another unsolved question, important for applications in the prime-number theory, concerns replaing theorems I and II by one-sided theorems. Counter-examples show that even theorem I cannot be replaced by one-sided theorem if only the positivity of the coefficients $b_{j}$ is required. It is not impossible, however, that for

$$
1+\sum_{j=2}^{n} z_{j}^{\prime \prime}
$$

2) Added in proof. Recently we showed with Vera T. Sós that the denominator $250(m+2 n)$ can be replaced by $104(m+n)$ and the new proof can dispense with Cartan's theorem. What is the best-possible constant instead of 104 we do not know. there exists a one-sided theorem. In a particular ease this problem has also been stated by $\mathrm{S}$. Ohowla in the following form: if $K$ is an arbitrarily large positive number and the number $s$ of the different integers $\lambda_{1}, \lambda_{2}, \ldots, \lambda_{s}$ exceeds a limit depending only upon $K$, then the minimum of the polynomial $\sum_{p=1}^{s} \cos \lambda_{p} x$ is less than $-\pi$.

The theorems $I$ and $I$, as regards their dependence upon $m$ and $n$, are nearly the best possible if $m$ is large, as one can see from suitable examples. They become not very exact, though not trivial, also in the case of $m=1, b_{1}=b_{2}=\ldots=b_{n}=1$; since also this case has an interesting application, as we shall see, it is worth-while to improve it. One can easily show that the inequality

$$
\frac{\left|\sum_{p=1}^{n} z_{\psi}^{t}\right|}{M_{2}(t)} \geqslant 1
$$

has an integer $t$-solution for $1 \leqslant t \leqslant n$, where equality ean be reached. Concerning $M_{1}(t)$ I can show that the inequality

$$
\frac{\left|\sum_{n=1}^{n} z_{v}^{t}\right|}{M_{1}(t)} \geqslant \frac{\log 2}{\frac{1}{1}+\frac{1}{2}+\ldots+\frac{1}{n}}
$$

has an integer $t$-solution with $1 \leqslant t \leqslant n$ and my late pupil $\mathrm{N}$. Sohweitzer showed the same solnbility of the inequality

$$
\frac{\left|\sum_{p=1}^{n} z_{v}^{t}\right|}{M_{1}(t)} \geqslant \frac{1}{2}
$$

in the interval $1 \leqslant t \leqslant 2 n$. The interesting question whether or not in the inequality (12) the right-side can be replaced by a constant independent of $n$ is left open in spite of the efforts of some mathematicians (P 126); an affirmative answer would be of significance in the approximative solution of algebraic equations.

Now I shail turn to the applications of the theorems mentioned above. They concern, at present, the theory of power-and Dirichlet-series with gaps, the theory of quasi-analytical functions, the approximative solutions of algebraic equations, differential equations, the approxmation by the translations of a function, inaugurated by $\mathrm{N}$. Wiener, various questions of the prime-number theory and of the zeta-function of Rie- 
mann. But before turning to them $I$ shall discuss another application, which shows most clearly the advantages of better localisation. It concerns the estimation of the $N$-number of real zeros of generalized trigonometrical polynomials

$$
f(x)=\sum_{\nu=0}^{n} c_{v} \cos \lambda_{\nu} x \quad \text { where } \quad 0=\lambda_{0}<\lambda_{1}<\ldots<\lambda_{n}
$$

and positive $o_{v}$-coefficients in an interval $(0<) a \leqslant x \leqslant a+a$. In the cuse of integer $\lambda_{y}$ we obviously have $N \leqslant(1+[d / \pi]) \lambda_{n}$, independently of the individual values of the $c_{y}$ and of $\lambda_{1}, \lambda_{2}, \ldots, \lambda_{n-1}$. To obtain a similar result in the case of arbitrary real $l$ 's we shall cover the segment $a \leqslant x \leqslant a+d b$ by a circle $\left|z-z_{0}\right| \leqslant r$ and estimate, instead of $N$, the number $N^{r}$ of zeros of $f(z)$ in the circle $\left|z-z_{0}\right| \leqslant r$. Applying the well-known estimation of Jensen we have

$$
N^{\prime}<\max _{\left|z-z_{0}\right| \leqslant s e r} \log \left|\frac{f(z)}{f\left(z_{0}\right)}\right| .
$$

The whole difficulty obviously lies in determining a $z_{0}$ - we may assume it as real - so that $\left|f\left(z_{0}\right)\right|$ should not be too small. Essentially, this can be done by Dirichlet's theorem. But then as regards $z=x_{0}, x_{0}$ being real, we can only give the following estimation: $1 \leqslant x_{0} \leqslant 100^{n}$; since the cirole $\left|z-x_{0}\right| \leqslant r$ must cover the segment $a \leqslant x \leqslant a+d, r$ must be chosen very large, about $(a+d) 100^{n}$, which spoils the upper estimation of

$$
\max _{\left|z \rightarrow x_{0}\right| \leqslant e r}|f(z)|
$$

considerably. Similarly, to use simply a covering circle around the origin leads to a too rough estimation. But using Theorem Ia, instead of Dirichlet's theorem, we can choose $x_{0}$ in $(a, a+\not h), i, e$. $a$ suitable $r$ is not greater than $d$, and although the lower estimation of $f\left(x_{0}\right)$ thus obtained is weaker, it is compensated by the advantage that a much better estimation of

$$
\max _{\left|x-x_{0}\right| \leqslant e^{r}}|f(x)|
$$

is offered in this way. We obtain the estimation performing the details

$$
N \leqslant(2 n+1) \log \frac{6(a+d)}{d}+3 d \lambda_{n}
$$

i. e. the estimation is again independent of the $o_{v}$ (apart from their positivity) and of the exponent $\lambda_{1}, \lambda_{2}, \ldots, \lambda_{n-1}$; however, it is dependent upon the number $n$ of terms, upon $a, i$. e. upon the position of our interval of length $a$. As the example $f(x)=\cos \lambda_{n} x$ shows, (13) is essentially the best possible. By the way, it would be an interesting task to investigater whether or not a similar theorem holds for arbitrary real coefficient and. also for the general polynomials (P 127)

$$
\left.\sum_{\nu=0}^{n}\left(a_{v} \cos \lambda_{v} x+b_{v} \sin \lambda_{v} x\right), \quad 0=\lambda_{0}<\lambda_{1}<\ldots<\lambda_{n}{ }^{3}\right) .
$$

We tum next to applications in the theory of power- and Dirichlet-series with gaps. First we sketch a proof of the classical gap-theorem of Fabry; this asserts that if

$$
f(z)=\sum_{n=1}^{\infty} A_{p} z^{\lambda_{\nu}}
$$

where the integer $\lambda_{v}$ satisfy Fabry's gap-condition $\lambda_{v} p \rightarrow \infty$ then every point of the convergence circle is a singular point. To prove this we need first the following theorem (which has been recently rediscovered and generalized in several directions by Bochner). Let

$$
g(z)=\sum_{=0}^{\infty} B_{v} z^{*}
$$

be regular in $|z|<1$ and in the sector $|z| \leqslant 1+\delta,|\operatorname{arc} z| \leqslant \delta$. Then there is a summation-matrix

$$
\begin{aligned}
& a_{00}, \\
& a_{10}, a_{11}, \\
& \ldots \ldots \\
& \sigma_{n 0}, a_{n 1}, \ldots, c_{n n}, \\
& \ldots \ldots \ldots
\end{aligned}
$$

dependent only upon $\delta$ such that in the domain $|z| \leqslant 1+\delta / 2,|\operatorname{arc} z| \leqslant \delta / 2$

$$
\left|g(z)-P_{n}(g)\right|=\left|g(z)-\sum_{\nu=0}^{n} c_{n_{\nu}} B_{\nu} z^{\nu}\right| \leqslant g^{n}
$$

for a suitable-positive $\vartheta=\vartheta(\delta, g)<1$. Further we observe that a simple application of theorem $I$ a shows that for arbitrary complex $C_{\text {v }}$-coefficients

$$
\max _{0 \leqslant x \leqslant 2 \pi}\left|\sum_{\nu=1}^{n} \partial_{\nu} e^{i \lambda_{r} x}\right| \leqslant\left(\frac{48 \pi}{\delta}\right)^{n} \max _{\alpha \leqslant x \leqslant \alpha+\delta}\left|\sum_{\nu=1}^{n} C_{\nu} e^{i \lambda_{\nu} x}\right| .
$$

3) Added in proof. A further theorem comparing $\mathbf{1}+\sum_{v=1}^{n} b_{y} z_{x}^{1}$ with its Bohr norm gives as an application that the number of zeros for $a \leqslant x \leqslant a+d$ in this general case is less than $9 d \lambda_{n}+\log (4 n+2)+2 n \log \left(10 \lambda_{n} / \delta\right)$ with $\delta=\min \left(\lambda_{p+1}-\lambda_{y}\right)$. It would be of interest to remove also the dependence upon $\delta$, if possible. 
In order to deduce Fabry's gap-theorem simply from (14) and (15), we may proceed as follows. If Fabry's gap-theorem were not true, then we might suppose without loss of generality that the circle of convergence of

$$
f(z)=\sum_{y=1}^{\infty} A_{\nu} z^{\lambda_{\nu}}
$$

with $\lambda_{\nu} / \nu \rightarrow \infty$ is the circle $|z|=1$ and $f(z)$ is regular for a sector $|z| \leqslant 1+\eta$, $|\operatorname{arc} z| \leqslant \eta$. Putting $z=\varrho e^{i x}$ in $P_{n}(f)$ (see $(14)$ ) we have

$$
P_{2_{n+1}}(f)-P_{\lambda_{n}}(f)=\sum_{i=1}^{n}\left\{\left(c_{\lambda,+1, \lambda_{\nu}}-c_{\lambda n, \lambda_{v}}\right) A_{v} e^{\lambda_{n}}\right\} e^{i \lambda_{\nu} x}+\left\{o_{\lambda_{n+1}, \lambda_{n+1}} A_{n+1} e^{\lambda_{n+1}}\right\} e^{i \lambda_{n+1} x} .
$$

Using (15) we obtain for $1-\eta / 2 \leqslant \varrho \leqslant 1+\eta / 2$

$$
\max _{|x|=e}\left|P_{\lambda^{n}+1}(f)-P_{\lambda_{n}}(f)\right| \leqslant\left(\frac{96 \pi}{\eta}\right)^{n+1} \max _{\substack{|z|=0 \\|\operatorname{ar} \delta| \leqslant \mid \leqslant \eta: 2}}\left|P_{\lambda_{n+1}}(f)-P_{\lambda_{n}}(f)\right| .
$$

But using (14) we obtain

$$
\begin{aligned}
\max _{\substack{|z|=g \\
|\operatorname{arc} z| \leqslant \eta_{i 2}}}\left|P_{\lambda_{n+1}}^{-}(f)-P_{\lambda_{n}}(f)\right| \leqslant & \max _{\substack{|z|=g \\
|\operatorname{arc} x| \leqslant \eta / 2}}\left|P_{\lambda_{n+1}}(f)-f(z)\right|+ \\
& +\max _{\substack{|z|=\mathrm{g} \\
|\operatorname{aro} g| \leqslant \eta / 2}}\left|P_{\lambda_{n}}(f)-f(z)\right| \leqslant \vartheta^{\lambda_{n+1}}+\vartheta^{\lambda_{n}}<2 \vartheta^{\lambda_{n}} .
\end{aligned}
$$

Thus from (16); using the gap-condition for the first time, we should obtain

$$
\max _{|z|=\varrho}\left|\boldsymbol{P}_{\lambda_{n+1}}(f)-P_{\lambda_{n}}(f)\right| \leqslant 2\left\{\vartheta\left(\frac{96 \pi}{\eta}\right)^{(n+1) ; \lambda_{n}}\right\}^{\lambda_{n}}<2\left(\begin{array}{c}
1+\vartheta \\
2
\end{array}\right)^{\lambda_{n}}
$$

for all $n>n_{0}, i . e$. the series

$$
\sum_{n}\left(P_{\lambda_{k+1}}(f)-P_{\lambda_{n}}(f)\right)
$$

would be uniformly convergent for $1-\eta / 2 \leqslant|z| \leqslant 1+\eta / 2$. But this would mean that $f(z)$ is regular in the circle $|z| \leqslant I+\eta / 2$, which is a contradiction already. The same method is applicable also to Dirichlet-series and yields the theorem that for the function

$$
h(s)=\sum_{n} D_{n} e^{-k_{n} s}, \quad k_{1}<k_{2}<\ldots,
$$

if $k_{n} / n \rightarrow \infty$ for $n \rightarrow \infty$, the points on its convergence-line are either all regularity-points or all singular-points of $h(s)$.

Another application concerns a theorem of Polya dealing with integral functions satisfying Fabry's condition. His result, a little vaguely expressed (a special case of which has been rediscovered by Zygmund and Marcinkiewicz) shows that an integral-function of finite order satisfying Fabry's condition has the same order and type in an arbitrarily small angle as on the whole plane. Now let

$$
f(z)=\sum_{p=1}^{\infty} a_{v} z^{\lambda_{z}}
$$

be an integral function satisfying Fabry's condition. A simple approximation process and suitable application of Theorem Ia yields the following general theorem.

Let $M(r, f)$ denote the absolnte maximum of $f(z)$ on $|z|=r$ and $M(r, f, \alpha, \beta)$ the absolnte maximnm of $f(z)$ on the arc $\alpha \leqslant \operatorname{arc} z \leqslant \beta$ of the circle $|z|=r$. Then for arbitrary $\varepsilon$ with $0<\varepsilon \leqslant 1 / 2$ there is an $r_{0}=r_{0}(f, \varepsilon, \beta-\alpha)$ such that for all $r>r_{0}$ we have the inequality

$$
M(r, f) \leqslant \frac{48 \pi}{\beta-a} M(2 r, f)^{8} M(r, f, \alpha, \beta) .
$$

Inequality $(17)$ is not trivial if $M(r, f)$ increases "not too quickly", and that is amply satisfied in the case of integral-functions of finite order. One easily obtains from it the above mentioned theorem of Pólya in a little strengthened form, and various theorems can also be obtained in this way, approaching a conjeoture of Pólya according to which for an integral-function $f(z)$ of finite order satisfying Fabry's gap-condition the relation

$$
\varlimsup_{r \rightarrow \infty} \frac{\log m(r, f)}{\log M(r, f)}=1
$$

Lolds (where $m(r, f)$ denotes the minimum of $|f(z)|$ for $|z|=r$ ).

Next I consider briefly an application in the theory of differential equations where the results, though very imperfect in form, seem to me to contain something new. We consider systems of the form

$$
\frac{d x_{v}}{d t}=\sum_{j=1}^{n} f_{v j}(t) x_{j}(t) \quad(v=1,2, \ldots, n) .
$$

The classical theorem of Poincaré, perfected by Perron, asserts that . if for suitable constants $a_{v i}$ we have

then

$$
\lim _{t \rightarrow+\infty} f_{v j}(t)=a_{v j}
$$

$$
\varlimsup_{t \rightarrow+\infty} \frac{1}{2 t} \log \left(\left|x_{1}\right|^{2}+\left|x_{2}\right|^{2}+\ldots+\left|x_{n}\right|^{2}\right)
$$

exists and is equal to one of the $n$ quantities $R \varrho_{v}$ where the $\varrho_{\text {v }}$ are the 
zeros of a certain characteristic equation of $n$-th degree. The significance of this result lies in the fact that with its help one can decide without knowing the explicit form of the solutions whether or not a slight change of the initial values changes the solutions "finally", $i$. e. for large $t$-value sometimes considerably; these are questions of stability. The question how Poinoare-Perron's theorem can be "finitised" is of interest in itself, but it can also be of importance, perhaps, in astronomy. First of all the interval $0<t<\infty$ must be replaced by a finite one, say $a \leqslant t \leqslant a+d, a$ being positive. Then the lim in the hypothesis must be replaced by an explicit estimation of the quantitie

$$
M_{v}=\max _{a \leqslant t \leqslant a+a}\left|f_{\nu}(t)-f_{\nu}(a)\right| \quad(y=1,2, \ldots, n) .
$$

Since in $\overline{\lim }$ we have a lower estimation of "big" values assumed by

$$
\sum_{v=1}^{\infty}\left|x_{v}(t)\right|^{2} \quad \text { for } 0<t<\infty
$$

therefore in the finitised form we expect a lower estimation of

$$
\max _{a \leqslant t \leqslant a+d} \sum_{p=1}^{n}\left|x_{\mu}(t)\right|^{2} \text {. }
$$

Owing to Theorem Ia this can actually be done if the quantities $M r_{v}$ are small and the estimation

$$
\max _{a \leqslant t \leqslant a+a} \sum_{\nu=1}^{n}\left|x_{\nu}(t)\right|^{2}>\frac{e^{2 l a}}{4 n^{2} d^{2}}\left(\frac{a}{6(a+d)}\right)^{2 n} \sum_{n=1}^{n}\left|x_{v}(0)\right|^{2},
$$

where $l$ denotes the minimal value of the above $R Q_{p}$, can be proved. What makes this result unsatisfactory is only the circumstance that, at present, very strong restrictions must be imposed on the $M_{p}$; ronghly speaking we must have essentially

$$
\sum_{x=1}^{n} M_{\eta}^{2}<d^{2 n} e^{-Q(a+d)}
$$

where $C$ depends only upon the system, not upon $a$ and $d, i$.e. not on the position of the interval $(a, a+d)$. It is very probable that $(20)$ holds essentially under the much weaker assumption that

$$
\sum_{\nu=1}^{n} M_{\nu}^{2}<C
$$

if $C$ sufficiently small but independent of $a$ and $d$ (P 128).

Next let us say a few words on quasi-analytioal functions. In a less precise and more general formulation, as usual, we shall deal with the question of characterising classes of functions in $a \leqslant n \leqslant b$ with the following property: if two functions of the class show "a similar behaviour in a neighbourhood of a $a$ " where $a<c<b$, then they are identical almost everywhere in $a \leqslant x \leqslant b$. The expression "similar behaviour in the neigh bourhood of $e$ " can be interpreted in different ways. I mention here only two possibilities. In the usual sense we shall deal with infinitely often differentiable functions; the "similar behaviour of $f_{1}(x)$ and $f_{2}(x)$ in 0 " means in this case that

$$
f_{1}^{(n)}(o)=f_{2}^{(n)}(o)
$$$$
(n=0,1, \ldots) \text {. }
$$

In this case the well-known theorem of Denjoy-Carleman states that the class of functions satisfying

$$
\max _{a \leqslant x \leqslant b}\left|f^{(n)}(x)\right|=M_{n}, \quad \text { where } \sum_{n=1}^{\infty} \frac{1}{\min _{v \geqslant n} \sqrt[n]{M_{y}}}=+\infty
$$

form such a quasi-analytical class. S. Mandelbrojt defined, for a fixed positive $e$, the "similar behaviour of the functions $f_{1}(x)$ and $f_{2}(x)$ in $c$ " by

$$
\lim _{h \rightarrow+0} e^{h-e} \int_{c-h}^{c}\left|f_{1}(t)-f_{2}(t)\right| d t<\infty
$$

where $a<0<b$, and he was able to characterise quasi-analytic classes in this sense for $a=0, b=2 \pi$ by expanding the functions into Fourier series and restricting the actually ocouring exponents. Now the Theorem Ia makes it possible to find "dual" theorems if we replace Mandelbrojt's definition by the slightly narrower one

$$
\overline{\lim }_{h \rightarrow+0} e^{h-e} \max _{c \rightarrow h \leqslant t \leqslant c}\left|f_{1}(t)-f_{2}(t)\right|<\infty .
$$

The functions of a quasi-analytical class either in the sense of (23) or in that of (24) are of course not necessarily derivable. One can also characterize quasi-analytical classes in the sense of (24) in the whole interval $(-\infty, \infty)$. Such a class is formed by all functions of the form

$$
f(x)=\sum a_{n} e^{i \lambda_{n} x}
$$

with the coefficient conditions

$$
\varlimsup_{\omega \rightarrow \infty} e^{(2 \omega \log \omega) / e} \sum_{n \rightarrow \infty}\left|a_{n}\right|<\infty .
$$

Thus we have found that all functions reprezented by series of the form (25) with restriction (26) form a quasi-analytical class in the sense of (24) for $(-\infty,+\infty)$. 
The next application concerns functions of the form

$$
G(z)=\sum_{v=1}^{n} a_{v} f\left(b_{v} z\right)
$$

where $f(z)$ is an arbitrary integral function of finite order, the $a_{y}$ and $b$, arbitrary complex numbers. As the example

$$
n=5, \quad b_{\nu}=c^{2 \pi i v / 5}, \quad a_{\nu}=1, \quad f(z)=\sum_{\nu=1}^{\infty} \frac{1}{\nu !} z^{5 \nu+1}
$$

shows, $G(z)$ can identically ranish, $i$. $e$. can be of smaller order than $f(z)$ itself. Such expressions as the one in (27) ocoured first in Wiener's theory of Tauberian theorems on functions of real variables. As regards functions of complex rariables, A. $O$. Gelfond has discussed the following interesting question: what are the necessary and sufficient conditions which the integral functiom $f(z)$ must satisfy in order that all function $F(z)$ regular for $|z| \leqslant R$ should be uniformly approximated by a $G(z)$ of the form (27), the $a_{v}$ and $b_{v}$ being chosen appropriately. The question of unicity of such an approximation leads in a natural way to the question of giving a lower estimation of $\max |G(z)|$ by $\max |f(z)|$, i.e. to the ques$|x|=r$

tion when $G(z)$ - which, of course, is always of not greater order than $f(z)$ - is of the same order as $f(z)$. As the example (28) shows, one cannot expect such result without any restriction upon $f(z)$. Theorem Ta makes it possible to characterise a general class of integral functions $f(z)$ of finite order such that $G(z)$ is an integral funetion of the same order as $f(z)$ itself if only $\sum_{n=1}^{n} a_{v} \neq 0$.

So far all applications have referred to Theorem I. We have mentioned that if $m=1$, then both theorem I and II become rather weak, and in the case of equal coefficients we have replaced those theorems, using another method, by a stronger estimation. Even the estimation belonging to Theorem II has an application in the approximative solution of algebraic equations. There is a well-known rule, the so-called rule of Graeffe-Bernoulli, according to which, having the equation

$$
f_{0}(x)=\sum_{p=0}^{n} a_{\nu} \dot{x}^{\prime \prime}=0
$$

to solve, we have to form the sequence:

$$
\begin{aligned}
& f_{1}(x)=f_{0}(\sqrt{x}) f_{0}(-\sqrt{x}), \\
& f_{p}(x)=f_{p-1}(\sqrt{x}) f_{p-1}(-\sqrt{x})
\end{aligned}
$$

and if

$$
f_{v}(x)=a_{0 v}+a_{1 v} x+\ldots+a_{n v} x^{n}
$$

$$
(\nu=0,1, \ldots)
$$

it has been asserted that denoting by $z_{1}$ one of the zeros of greatest absolute value we have

$$
\left|z_{1}\right|=\lim _{p \rightarrow \infty}\left|\frac{a_{n-1, v}}{a_{n v}}\right|^{12^{p}} .
$$

This is actually true if $z_{1}$ is the zero with the greatest absolute value, but - though never explicitly mentioned, not even in Runge's Enzyklopädie-article - false in general. Ostrowski gave in 1940, in a very thorough paper in Aota Mathematica, a modification of the rule of Graeffe-Bernoulli by means of which he was able also to replace the lim in (31) by an inequality for all $\boldsymbol{\nu}$-values. His idea, essentially, was to correspond to the coefficients of $f_{v}(x)(n+1)$ other valnes by a certain polygon-construction and to work with those. Now the above-mentioned form of Theorem II makes it possible to save the rule in another way. Among the many possibilities I mention here only one. Let us form again the $\boldsymbol{v}$-th Graeffe-transform

$$
f_{\nu}(x)=a_{0 \nu}+a_{1 \nu} x+\ldots+a_{n \nu} x^{n}, \quad a_{n \nu}=1,
$$

and with these coefficients determine the quantities $s_{1}, s_{2}, \ldots, s_{2 n}$ sinocessively by the Newton-Girard formulae

$$
\begin{aligned}
& s_{1}+a_{n-1, n}=0 \\
& s_{2}+a_{n-1, n} s_{1}+2 a_{n-2, y}=0 \text {, } \\
& \text {........................... } \\
& s_{2 n}+a_{n-1, v} s_{2 n-1}+\ldots+a_{1 v} s_{n+1}+a_{01} s_{n}=0 \text {. }
\end{aligned}
$$

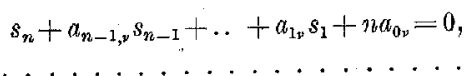

Then the maximum of the quantities $\left|s_{j}\right|^{1 / j}(j=1,2, \ldots, 2 n)$ will be the approximative value $\left|z_{1}\right|^{2^{p}}$; more exactly we have

$$
\left(\frac{1}{n}\right)^{2^{-\nu}} \leqslant \frac{\left|z_{1}\right|}{\left(\max _{j=1, \ldots, 2 n}\left|s_{j}\right|^{1 / 2}\right)^{2^{-\nu}}} \leqslant 2^{2^{-\nu}} .
$$

We have already mentioned the conjecture that the above-mentioned stronger form of Theorem IT for equal coefficients and $m=1$ is not the strongest. If this were true, it would have an important application here, since then $\max \left|s_{j}\right|^{1 / 3}$ would be replaced by $\max \left|s_{i}\right|^{1 / j}$, which would resince then $\max _{j=1, \ldots, 2 n}\left|s_{j}\right|^{1 / 3}$ would be replaced by $\max _{j=1, \ldots, n} \mid s_{j}$ 
duce the calculations very considerably. By the way, we notice that all necessary computations are built up from the four fuudamental operations and the extraction of roots; hence the above theorem can perhaps serve as the theoretioal basis for a computing machine solving algebraic equations.

Finally we turn to applications which are perhaps the deepest, and use Theorem II. They concern the distribution of prime-numbers and the theory of zeta-function of Riemann. As is well known. Riemann introduced in 1859 , essentially for the proof of the prime-number theorem conjectured by Gauss and Legendre, the function $\zeta(s)$ of the complex variable $s=\sigma+i t$, defined for $\sigma>1$ by the series

$$
\zeta(s)=\sum_{p=1}^{\infty} \frac{1}{n^{s}} .
$$

as I have mentioned. The paper of Riemann consists only of 9 pages, but its influence is as great now as it was at the end of the last century, when Hadamard was able to make the first steps to prove some of the assertions stated in it without a rigorous proof. Now all of his unproved assertions are proved except one. This, the famous conjecture of Riemann, asserts, as I mentioned formerly, that the function $\zeta(s)$ (which can be analytically continued over the whole plane and has only one pole at $s=1$ ), does not vanish in the half-plane $\sigma>1 / 2$. This conjecture has been a constant challenge to all analysts till now, and advance can be made only by the heaviest weapons. Hilbert said once that if he slept for 500 years and then awakened, he would first ask is Riemann's conjecture solved or not. Also the papers left by Riemann show how much effort lies behind the few lines he has written on this subject. Some of the assertions of his paper have turned out to be false, e. $g$. he asserted at the end of his paper that the number $\pi(x)$ of the prime-numbers not exceeding $x$ is always less than the Gaussian approximative-value $\int_{2}^{x} d v / \log v$, and after Erhard Schmidt's first results Littlewood disproved it in 1914. Perhaps this is one of the reasons why some analysts have doubts as to the correctiness of the conjecture. Anyway, it is known that the strip $0<\sigma<1$ contains an infinity of zeros (so called non-trivial zeros) of $\zeta(s)$ and they lie symmetrically to the point $s=1 / 2$; this means that no half-plane $\sigma>\vartheta$ for $\vartheta<1 / 2$ oan be free of zeros. Thus the proof of Riemann's conjecture would also mean that all non-trivial zeros lie on the line $\sigma=1 / 2$.

Now, in the investigation of the question there are five trends; to some of them $I$ have nothing to add. The first consists in estimating the number of zeros in different subdomains of the strip $1 / 2<\sigma<1$. After the first results of Bohr, Landau and Carlson, the sharpest results have been reached by Ingham; to give an idea of them I mention only that denoting by $N(\alpha, T)$ the number of non-trivial zeros of $\zeta(s)$ in the domain $\sigma>a, 0<t \leqslant T$, he shows, for a fixed $\alpha$ where $5 / 8 \leqslant \alpha \leqslant 1$ and $T \geqslant 2$, the estimation

$$
N(\alpha, T)<c_{1} T^{8(1-\alpha) / 3} \log ^{5} T
$$

with a numerical $o_{1}$. This - together with a result to be mentioned later with a low same sort but valid for $1 / 2 \leqslant a \leqslant 5 / 8-$ and with another estimation of the same sort but valid for $1 / 2 \leqslant a \leqslant 5 / 8-$ has been of great importance in the theory of numbers, for it has enabled Ingham to prove a theorem which 25 years ago seemed entirely impos sible, namely that between $n^{3}$ and $(n+1)^{3}$ there is a prime-number form - the estimation

$$
N(a, T)<r_{2} T^{9(1-a) / 4} \log ^{5} T .
$$

This and (35) shows anyway, that the estimation is more difficult near the line $\sigma=1$. As Ingham has remarked further, one could even prove that for each fixed positive $\varepsilon$ and $n>n_{0}(\varepsilon)$ there is a prime between $n^{2+s}$ and $(n+1)^{2+5}$ if, instead of $(35)$ or $(36)$, we had for $1 / 2 \leqslant \alpha \leqslant 1$ the estimation

$$
N(\alpha, T)<\rho_{3} T^{2(1-\alpha)} \log ^{5} T
$$

where $\theta_{3}$ - and later $\theta_{4}, \ldots$ - are all numerical constants. Now Theorem II has made it possible to prove (37) essentially near $a=1$, more exactly to prove the existence of a $a_{4}$, where $1 / 3<0_{4}<1$, such that for $c_{4} \leqslant a \leqslant 1$ we have

$$
N(\alpha, T)<c_{5} T^{2(1-a)+600(1-\alpha)^{101 / 100}} \log ^{6} T .
$$

It is interesting to note that our method works in the most critical region; here it constitutes the best estimation at present ${ }^{4}$ ).

The proof of this, as well as the remaining applications, is rather complicated; for lack of time I have to confine myself to indicating how Theorem II will be applied. We have a rational function of the form

where

$$
U(z)=\sum_{n=1}^{n} \frac{1}{z-z_{n}},
$$

$$
\left|z-z_{1}\right| \leqslant\left|z-z_{2}\right| \leqslant \ldots \leqslant\left|z-z_{n}\right|
$$

4) Ldded in proof. After some improvements the right side can be replaced by $7^{2(1-a)+(1-n d)^{1,14}}$ 
and a real $m$ much greater than $n$. Then we have, for an integer $k$ with $m \leqslant k \leqslant m+n$, the inequality, $i, e$. the $k$-th derivative of $U(z)$ for a suitable $t$ from a "small" interval can be estimated from below by masimal term alone.

The second trend consists in finding in the strip $0<\sigma<1$ possibly large domains without zeros of $\zeta(s)$. In this respect the results are rather poor at present. U'sing the ideas of Littlewood and Landan and recent estimations of Vinogrador on trigonometrical sums, one can show the existence of a $c_{5}$ such that $\zeta(s)$ does not vanish in the domain

$$
\sigma>1-\frac{\theta_{6}}{(\log t \log \log t)^{3 / 4}}, \quad t>a_{7} .
$$

This result is also a very important tool in the proof of Ingham's theorem. To this field I have nothing to add at present. Nevertheless $I$ think it very probable that Theorem II will give results also in this field; I mentioned it only as an introduction to the third trend. By this we see that we are not moderate if we confine our attention to the so-called quasi-riemannian oonjecture which asks ouly the existence of a $\vartheta$ with $1 / 2 \leqslant \vartheta<1$ such that $\zeta(s)$ has in the half-plane $\sigma>2$ at most a finite number of zeros. As a matter of fact, for most applications the validity of this weaker hypothesis would be sufficient. The third trend tries to find equivalent formulations for Riemann's conjecture; we try to do that only for the quasi-riemannian conjecture. Theorem II has made it possible to show that the quasi-riemannian hypothesis is completely equivalent to the following. There are an $\alpha$ and $\beta$ where $\alpha \geqslant 2,0<\beta \leqslant 1$, such that for

$$
t>c_{3}, \quad t^{\alpha} \leqslant \frac{N}{2} \leqslant N_{1}<N_{2} \leqslant N
$$

we have

$$
\left|\sum_{N_{1} \leqslant p \leqslant N_{2}} e^{i t \log p}\right|<c_{9} \frac{N \log ^{10 n} N}{t^{\beta}} .
$$

If $N$ is greater than $o^{t^{10}}$, it is easy to prove this inequality. What is surprising in this result, is this. Replace in (40) the quantities $\log p$ by $\log ^{0,99} p$ or $\log ^{1,01} p$ respectively. Then the corresponding inequalities can be proved in an elementary way, with the aid of Vinogradov's really ingenious method. The same is true when we replace $\log p$ by $\log p(\log \log p)^{0,01}$ and $\log p(\log \log p)^{-0,01}$ and so on.

The fourth trend refers to the mysterious connection between primes and non-trivial zeros of $\zeta(s)$. Let us suppose that the half-plane $\sigma>\vartheta$ is the "argest" half-plane without zeros of $\zeta(s)$, roughly speaking. Then it is known that

$$
|\Delta(x)|=\left|\pi(x)-\int_{2}^{x} \frac{d v}{\log v}\right|<c_{00} x^{\theta} \log x .
$$

Now the question arises whether or not a better estimation of $\{\Delta(x)\}$ can be derived. The answer, as Phragmén showed, turns out to be negative, $i$. e. the abscissae of the "greatest" half-plane containing no zeros and the "smallest" exponent $\vartheta$ in (41) are found to be equal. As a matter of fact, we do not know at present any $\vartheta<1$ with this property; at present we know only the estimation given in (39) and what we can hope is to diminish the exponent $3 / 4$ in it. From the hypothesis that $\zeta(s)$ does not vanish in a domain

$$
\sigma>1-\frac{\theta_{11}}{\log ^{\gamma} t}, \quad t \geqslant 2,
$$

for a fixed $0<\gamma \leqslant 1$ we can deduce only the estimation

$$
|\Delta(x)|<x e^{-c_{12} \log 1 /(1+\gamma)} x .
$$

It has been a long-standing question, particularly important in the case, when even the quasi-riemannian hypothesis is false, whether or not a better estimation of $|\Delta(x)|$ can be derived from (42). Theorem II enables us to decide also this question negatively, i. e. from (43) one can actually deduce the non-vanishing of $\zeta(s)$ in the domain (42). Hence the exaat connection between the remainder-term $\left|\Delta\left(x_{i}\right)\right|$ and the domain of non-vanishing of $\zeta(s)$ is found also for this case.

The fifth trend line $\sigma=1 / 2$. But since my method has nothing to do in this direction, I do not go into it in detail.

The last application I am going to speak about deals also with the remainder-term $|\Delta(x)|$ and I shall only touch it. As I have mentioned, the knowledge of the "largest" half-plane $\sigma>\vartheta$, not containing zeros of $\zeta(s)$, determines the order of $|\Delta(x)|, i . e$. infinitely often we have

$$
\left|\Delta\left(x_{p}\right)\right|>w_{v}^{\theta-s}
$$

But the former method is purely an existence-proof, gives no information about the $x_{y}$ and is indirect. As Littlewood has remarked, all those disadvantages would be eliminated if the lower estimation of $|\Delta(x)|$ could be made dependent not upon the largest half-plane not containing a zero, about which we know nothing definite, but upon a single zero of $\zeta(s)$. 
Theorem Ir enables us also to prove Littlewood's desideratum and to prove the following theorem: if $\varrho_{0}=\beta_{0}+i \gamma_{0}$ for $\beta_{0} \geqslant 1 / 2$ is a non-trivial zero of $\zeta(s)$, then for $T>c_{13}$ and $T>e^{e l \rho_{0}}$ we have

$$
\max _{1 \leq x \leq T}|A(x)|>T^{60} e^{-c_{14} \frac{\log T \log \log \log T}{\log \log T} .}
$$

Here $\theta_{13}$ and $o_{14}$ are numerical constants whose values oan be given explicitly. To get finer results in this way we should need the one-sided refinement of our theorems mentioned in the first part.

The list of applications is still incomplete. But perhaps those discussed above already show that the way of interpretation of Dirichlet's and Kroneoker's theorems which we have systematically followed, is a fruitful one. I hope I have also succeeded in showing that this theory is at the very beginning of its development and many more applications oan be expected.

\section{ON A THEOREM OF F. AND M. RIESZ}

BY

HEN RY HELSON ${ }^{1}$ ) (NEW HAVEN, Conn.)

The theorem in question is the following ([3], or [4], p. 157-158): Let

$$
f\left(r e^{i x}\right)=\sum_{n=0}^{\infty} a_{n} 2^{n} e^{i n x}
$$

be an analytio function defined in the unit oirole, and suppose that

$$
\int_{0}^{2 \pi}\left|f\left(r e^{i x}\right)\right| d x
$$

is bounded for $r<1$. Then there is a summable funotion $f_{0}\left(e^{i x}\right)$ defined on the boundary of the oirole and summable, suoh that

$$
\lim _{r \rightarrow 1} \int_{0}^{2 \pi}\left|f_{0}\left(e^{i x}\right)-f\left(r e^{i x}\right)\right| d x=0
$$

and the Fowier series of $f_{0}$ is

$$
f_{0}\left(e^{i x}\right) \sim \sum_{n=0}^{\infty} a_{n} e^{i n x}
$$

The statement of the theorem and its original proof are function-theoretic. The purpose of this note is to give a new proof from a different point of view, which is closer to the spirit of some of the applications of the theorem ${ }^{2}$.

We shall have to consider bounded complex-valued measures defined on the field of Borel subsets of the interval $(0,2 \pi)$. Associated with such a measure $\mu$ is a function of bounded variation on the interval defined by

$$
\mu(x)=\mu\left(I_{x}\right),
$$

where $I_{x}$ is the set of $y$ satisfying $0<y \leqslant x$. It will be clear from the context whether a symbol is being used to denote a measure or the corresponding function of bounded variation.

1) Jewett Fellow of the Bell Telephone Laboratories.

2) The author expresses lis indebtedness to Dr. S. Kakutani and Dr. J. Wermer for conversations about the subject of this note.

Colloguium Mathematieum III. 2 